\title{
The Design and Development of MOVE-IT: A System for Remote Vestibular and Oculomotor Assessment in People with Concussion
}

\author{
Selena HILL ${ }^{\mathrm{a}, \mathrm{b}, 1}$ Christopher BARR $^{\mathrm{a}}$ Maggie KILLINGTON ${ }^{\mathrm{a}, \mathrm{b}}$ \\ James MCLOUGHLIN ${ }^{\mathrm{a}}$ Rory DANIELS ${ }^{\mathrm{c}}$ and Maayken VAN DEN BERG ${ }^{\mathrm{a}}$ \\ ${ }^{\mathrm{a}}$ Flinders University, College of Nursing and Health Sciences \\ ${ }^{b}$ SA Health, South Australian Brain Injury Rehabilitation Service \\ ${ }^{\mathrm{c}}$ Flinders University, College Science and Engineering
}

\begin{abstract}
Background: Dizziness is one of the most common symptoms following concussion and requires a thorough vestibular assessment. However, due to limited tools and evidence on remote vestibular assessment and intervention, people unable to attend in-person consults cannot receive effective care. Objective: This study aims to describe the design and development process of MOVE-IT. MOVE-IT is a mobile phone application with an associated head mount device and clinician dashboard which aims to enhance vestibular assessments and intervention via telehealth by enabling clinicians to clearly observe client's eye movements. Methods: This study used a Living Labs methodology including the use of a scoping review, user engagement, multi stakeholder engagement, real-life settings, and co-creation. MOVE-IT was developed in three phases: Exploration, Experimentation and Evaluation. This paper describes the Exploration and Experimentation process. Exploration included a scoping review, focus group and consultation interviews. Experimentation included the co-creation of a minimum viable product in a real-life setting with regular feedback from multi-stakeholders. Outcome: MOVE-IT includes three components: a mobile phone application, head mount device and clinician dashboard. MOVE-IT aims to enhance the use of telehealth for vestibular assessments by: (1) using the head mount device to enable video recording of client's eyes during assessment, (2) allowing clinicians to view client's eye movements via the clinician dashboard whilst (3) a support person assists in the physical aspect of the vestibular assessment by a step-by-step guided video in the mobile application. Conclusion: The Living Lab method was a useful strategy for developing MOVE-IT. MOVE-IT meets all predefined functionality requirements and potentially provides a solution for remote vestibular assessment and intervention in the concussion population. The Evaluation phase will be conducted next to test usability, reliability and validity of MOVE-IT.
\end{abstract}

Keywords. Vestibular Rehabilitation, telehealth, mobile application

${ }^{1}$ Corresonding author: Selena Hill, Flinders University, College of Nursing and Health Sciences/ SA Health, South Australian Brain Injury Rehabilitation Service, Adelaide, South Australia, Australia; E-mail: hill0451@flinders.edu.au 


\section{Introduction}

Concussion is a mild traumatic brain injury (TBI) and is responsible for an estimated 70$85 \%$ of TBI worldwide [1]. Although it is widely recognised that concussion is underdiagnosed, the projected global incidence lies between 40 and 55 million per year [1]. Most people with concussion recover within two weeks without requiring specialist input [2]. However, approximately $10-15 \%$ of individuals experience persistent symptoms 10-14 days after the impact [2]. Dizziness is considered one of the most common symptoms following concussion and a risk factor for prolonged recovery [2-4]. A thorough vestibular assessment is required for these individuals, which involves identifying abnormal eye movements during vestibular testing [3, 4].

During the COVID-19 pandemic, telehealth services were temporarily added to the Medicare Benefits schedule by the Australian Government to maintain physical distancing whilst providing health care services [5]. During this time, there was a rapid increase in the use of telehealth in tertiary Australian hospitals $(2,255 \%)$, along with primary care physicians to continue providing individuals with health care services when in-person consults were not available $[5,6]$. Objective vestibular assessments are challenging to administer using telehealth and there is currently a paucity of literature on individualized vestibular assessment and rehabilitation delivered via telehealth. A significant limiting factor in this area is the inability to clearly observe the client for abnormal eye movements during assessment.

Mobile phones are very commonly used currently in society and various mobile applications have been developed to successfully provide low-cost solutions to assist with health care interventions [7]. Although very few have investigated the use of mobile applications in vestibular assessment and rehabilitation [8-11].

This paper aims to describe the design and development process of MOVE-IT (Management of OculoMotor and Vestibular Evaluation, Intervention and Treatment). MOVE-IT is a mobile phone application with associated head mount device and clinician dashboard which aims to enhance vestibular assessments via telehealth by enabling clinicians to clearly observe client's eye movements during vestibular testing. A mobile application was used, as the general population commonly have access to a mobile device and, when used in combination with a head mount device, it allows free movement of the head and body during vestibular assessments and rehabilitation. MOVE-IT also aims to improve compliance and reliability of maneuver interventions without face-to-face contact with a clinician. This provides potential to progress vestibular rehabilitation at home more effectively.

\section{Methods}

\subsection{Overview}

A multi-method approach was used to develop MOVE-IT including a scoping review (manuscript in preparation for publication), a focus group, consultation interviews and a minimum viable product. 


\subsection{Methodology}

This study used a Living Lab methodology to enable the creation of a mobile application, associated clinician dashboard and head mount device. The Living Lab methodology focuses on inter-professional collaboration to integrate technical innovations into health interventions [12]. The principles of the Living Lab methodology include co-creation, active user involvement, real-life experimentation, multi-stakeholder and multi-method approaches (Figure 1.) [13]. The phases of the innovation process include exploration, experimentation, and evaluation [13]. The focus on multi-stakeholder engagement in the development process provides researchers and developers with immediate user feedback, improving the likelihood of a successful and sustainable innovation [14]. The study was nested within a larger study evaluating feasibility and effectiveness of an interdisciplinary clinic for people with mild TBI. This study was approved by the Central Adelaide Local Health Network Human Research Ethics Committee (HREC/18/CALHN/365) and funded by Lifetime Support Authority.

\begin{tabular}{|l|}
\hline $\begin{array}{c}\text { Multi-Method } \\
\text { Approach }\end{array}$ \\
\hline - Living Lab \\
methodology \\
- Literature \\
review \\
- Focus group \\
- Consultation \\
Interviews \\
- Minimum \\
viable product \\
- Prototype \\
testing \\
- Usability testing \\
\hline
\end{tabular}

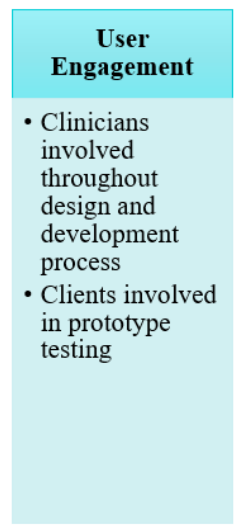

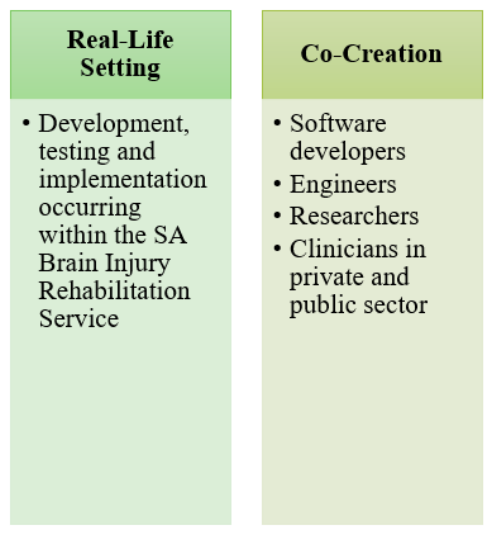

Figure 1. Principles of the Living Lab methodology.

\subsection{Exploration}

\subsubsection{Scoping review}

A scoping review (manuscript in preparation for publication) was conducted to evaluate the literature for technologies to assist in remote assessment and intervention of vestibular and oculomotor impairments. We searched five relevant databases for studies published in English up to June 2020. Three search strings were used for "Vestibular" AND "telehealth OR mobile app" AND "Rehabilitation". Two independent reviewers completed initial screening and disagreements were resolved by a third reviewer. Only peer-reviewed journals including interventions able to be performed independently or with remote assistance from a clinician were included.

\subsubsection{Focus group \& Consultation interviews}

Next, a focus group was held to establish the need for a device to assist with remote assessment of vestibular impairment and the necessary functionalities to be included within the system. During the design and development stage, clinicians were consulted 
as the primary users, as the purpose of the system is to allow a range of specific vestibular and oculomotor tests to occur remotely. The focus group included two occupational therapists and a neurological physiotherapist experienced in rehabilitation of mild TBI. The aim was to establish minimum requirements of MOVE-IT with consideration of minimising potential barriers to usability. Consultation interviews were also conducted with another five neurological physiotherapists and an orthoptist with experience in mild TBI rehabilitation.

\subsection{Experimentation}

Co-creation was implemented through collaboration with the development team - a team of future users, researchers, engineers, and software developers. The development team collaborated to translate the ideas and minimum requirements from the focus group and consultation interviews into an innovative technology design - MOVE-IT. Weekly meetings occurred between the development team to provide updates on MOVE-IT, to allow feedback throughout the design and development process. A minimum viable product was produced and provided to future users for testing and feedback on the usability of the device. Software developers and engineers encouraged users to trial the device and report any bugs or issues that occurred during testing. This feedback was then taken back to the development team, adjustments were made, and MOVE-IT was updated and provided back to future users for another round of testing. Once a draft prototype has been established, client's input will be sought as users in prototype testing, to assess ease of use and understanding as well as comfort of the head mount device.

\subsection{Evaluation}

The evaluation phase is planned for January-June 2021 and will involve clients and clinicians testing the accuracy, validity and usability of MOVE-IT in vestibular and oculomotor assessment and interventions via telehealth in the concussion population. This phase will aim to (1) evaluate if clients are able to correctly follow the guided assessment to produce valid data, (2) determine the accuracy of the data, and (3) whether diagnosis made using the MOVE-IT app provides the same results as in person assessments. Usability will also be assessed in terms of ease of use, ease of understanding and comfort of the device.

\section{Outcome}

In the following, we present the results of the exploration and experimentation phases of the design and development process of MOVE-IT.

\subsection{Scoping Review}

The full scoping review will be presented elsewhere. In summary, the study results demonstrated that research on the use of telehealth literature to enable remote assessment and intervention of vestibular disorders is limited. Moreover, no tools were identified to perform a remote vestibular assessment while enabling a clear view of client's eyes. 


\subsection{Focus group \& Consultation Interviews}

Findings from the focus group and consultation interviews reported the inability to clearly observe client's abnormal eye movements as the biggest barrier to remote vestibular and oculomotor assessment and intervention. Also, the maneuvers required for assessment and intervention are difficult to explain to the client or person assisting. Minimum requirements for the head mount device, mobile application and clinician dashboard were discussed with the aim to overcome any anticipated barriers and reduce issues with usability. A full list of inclusions is presented in Table 1. Additionally, a record of assessments and Canalith Repositioning Maneuvers (CRMs) was produced that clinicians wished to successfully perform remotely, requiring a clear view of client's eye movements.

Table 1. Focus Group \& Consultation Interview Results - Functionality Requirements of MOVE-IT

\begin{tabular}{|c|c|c|}
\hline Head Mount Device & Mobile Application & Clinician Dashboard \\
\hline Low cost & Include $\quad$ all $\quad$ required & Required to meet SA \\
\hline Easily reproducible & $\begin{array}{l}\text { assessments and CRMs } \\
\text { Demonstration video with } \\
\text { audio voice over instructions } \\
\text { prior to commencement of } \\
\text { assessments and CRM }\end{array}$ & $\begin{array}{l}\text { Health security standards } \\
\text { Ability to re-watch videos } \\
\text { an unlimited number of } \\
\text { times }\end{array}$ \\
\hline $\begin{array}{l}\text { Not interfere with } \\
\text { head position during } \\
\text { Benign Paroxysmal } \\
\text { Positional Vertigo } \\
\text { (BPPV) CRMs }\end{array}$ & $\begin{array}{l}\text { Ability to re-watch } \\
\text { demonstration videos before } \\
\text { attempting assessment or CRM }\end{array}$ & $\begin{array}{l}\text { Simultaneous video of } \\
\text { client's eyes with } \\
\text { demonstration video to } \\
\text { assess at what point during } \\
\text { assessments abnormal eye } \\
\text { movements occurred }\end{array}$ \\
\hline $\begin{array}{l}\text { Light weight to reduce } \\
\text { discomfort to the neck }\end{array}$ & $\begin{array}{l}\text { Video and audio instructions } \\
\text { during assessments and CRMs }\end{array}$ & $\begin{array}{l}\text { Ability to add tags for ease } \\
\text { of search of videos }\end{array}$ \\
\hline $\begin{array}{l}\text { Clear video of the eyes } \\
\text { during head } \\
\text { movements }\end{array}$ & Simple to navigate & \\
\hline $\begin{array}{l}\text { Ability to hold a range } \\
\text { of mobile phone types }\end{array}$ & Available on android or IOS & \\
\hline $\begin{array}{l}\text { Ability to see both } \\
\text { eyes once mobile } \\
\text { device is attached }\end{array}$ & $\begin{array}{l}\text { Cloud storage to enable ability } \\
\text { to send videos securely to } \\
\text { clinicians }\end{array}$ & \\
\hline
\end{tabular}

\subsection{MOVE-IT}

The solution to the identified challenges with vestibular assessments and intervention when using telehealth led to the innovative concept materialized as MOVE-IT. MOVEIT is a three-part system: a mobile phone application, head mount device and clinician dashboard. The mobile phone application was developed in combination with a lightweight 3D printed head mount device which enables video recording of client's eye movements throughout vestibular testing. This allows the client to move their head and body in all directions for various vestibular assessments and CRMs whilst still enabling a clear video recording of the client's eyes. The mobile application directs the support person, such as a family member or friend, with a step-by-step video-guided tutorial, to perform the vestibular and oculomotor assessments. Using the application during the 
telehealth session allows the clinician to indicate which assessment should be performed and observe or guide the process to ensure accuracy of the assessment technique. The video of the client's eye movements, recorded during the remote consultation assessment, is then sent to the clinician dashboard for diagnosis, and interpreted by the clinician. This enables a higher resolution video compared to real-time video such as telehealth.

\subsubsection{MOVE-IT mobile application}

To ensure a user-friendly mobile device interface, a minimalist layout was used, as well as a clear step-by-step process to guide users through assessments and CRMs. Due to difficulties instructing the support person to assist with the assessments, initial video and audio voice over demonstration was included, followed by guided video and audio instructions during the assessment. A secure cloud-based storage system was used to allow clients to efficiently send high resolution videos to the clinician, accessible through the clinician dashboard. The mobile application was developed for both IOS and Android to capture both types of devices.

The mobile application begins with a log in and registration page. Once logged in the individual can select the appropriate assessment or CRM as advised by their clinician via telehealth. The mobile application will provide a short video demonstration of the assessment or CRM with option at the end to repeat the video or begin testing. A safety warning appears prior to each assessment or CRM advising users of possible symptom provocation. The mobile phone is then placed in the head mount device and lined up to achieve a clear picture of the client's eyes (Figure 2.) (in the case of an assessment) or comfortably in the middle of the forehead (in the case of a CRM). The support person follows the step-by-step guided tutorial and the clinicians remotely view the process via telehealth to ensure accuracy of assessment or CRM. The video is then sent to the clinician dashboard for remote viewing.

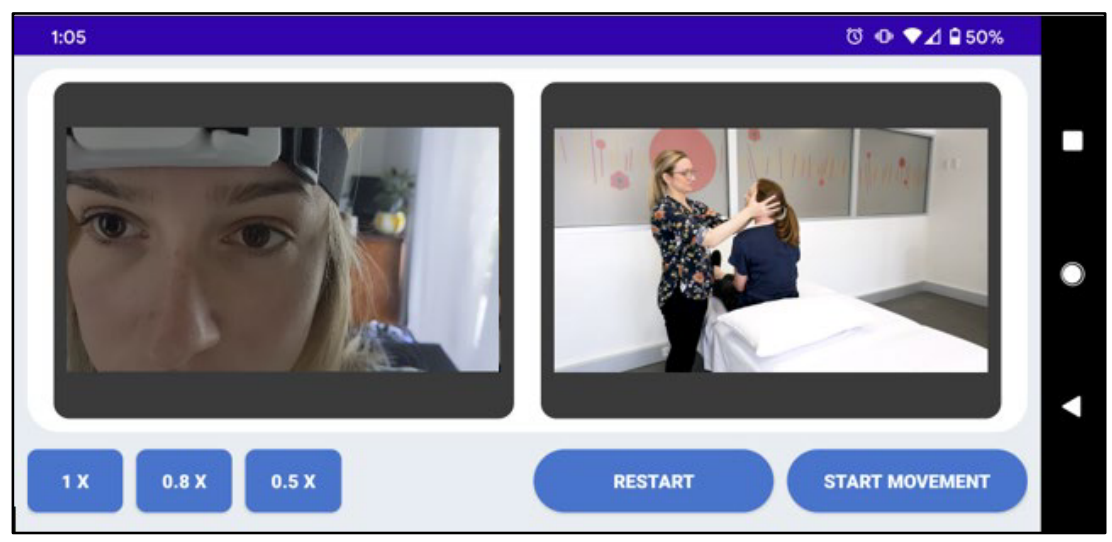

Figure 2. Screenshot of mobile application. Video recording of client's eyes (left), guided video tutorial (right).

\subsubsection{Clinician dashboard}

The clinician dashboard is accessed through a secured website with an email and password $\log$ in. Clinicians are notified by email when a client has uploaded a new video 
to the dashboard. Clinicians can repeatedly view videos uploaded by their clients to facilitate diagnosis. The eye movement video and corresponding assessment procedure video are displayed in parallel to enable clinicians to determine at what times abnormal eye movements occurred. The clinician can add 'tags' to videos to enhance ease of access to search videos later for reassessment.

\subsubsection{Head mount device}

With respect to the requirements collated in the focus group and consultation interviews, a lightweight head mount device was designed (117 grams) and 3D printed, resulting in easy postage and minimal discomfort to the neck, whilst minimising the cost to reproduce. The head mount device was developed in such way that it can hold a large range of phones, enabling a clear view of both eyes, keeping the front screen visible for the assisting support person. Two head mount device attachments were developed: one included an extended arm to allow a clear view of both eyes, the other sits flat on the head to enable minimal interference during CRMs (Figure 3.). User feedback was incorporated throughout the design and development process. Three prototypes were developed with improvements on size and stability each time following user feedback.

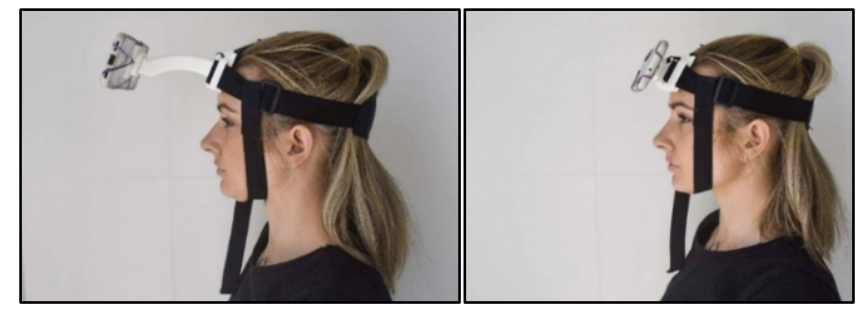

Figure 3. MOVE-IT long arm head mount device on left, short arm head mount device on right.

\section{Discussion}

In this paper we described the development of MOVE-IT, a three-part system designed to enable remote vestibular assessment following concussion via telehealth using the Living Lab methodology. The Living Lab methodology was used to improve the prospect of a fit for purpose system. Co-creation was achieved within the development team and regular consultation with multi-stakeholders. Users were involved throughout the design and development process in the setting of a mild TBI service, regularly using telehealth. Clients were not included in the design phase, as the issue focused on overcoming challenges clinicians were experiencing with objective assessment via telehealth. Clients feedback will be incorporated following usability testing of the prototype and subsequent modifications will be made as necessary.

To our knowledge, this is the first low-cost device that can enable a clear view of client's eyes during a remote vestibular assessment when using telehealth. This innovative concept enables clients to receive specialist vestibular input even if they are unable to access in-person consults. Telehealth has been used as a tool to manage concussion in rural areas, although a commonly reported inhibiting factor is limitations in physical assessment [15]. Dizziness is one of the most common symptoms following concussion and requires objective assessments to view abnormal eye movement [2-4]. 
The Vestibular Oculomotor Screening (VOMS) tool has been performed over telehealth [16] as it relies on subjective symptom reporting however, should not be used in isolation because of limitations related to inter-tester reliability, the possibility of an anxiety component related to dizziness and recall bias [3]. MOVE-IT allows clinicians to view client's abnormal eye movements through objective testing with the use of the head mount device and mobile phone camera. Concurrently, a support person assists the client with the physical component of the vestibular assessment via a guided video tutorial on the mobile application.

MOVE-IT is designed to be implemented by posting the head mount device to clients once they are referred to the service. The clients then download the MOVE-IT mobile application to their personal mobile device. The head mount device and mobile application would then be used during a telehealth consult under the guidance of a clinician to select required objective tests. A support person will assist the client by following the guided video tutorial to perform the physical aspect of the assessment, whilst the clinician supervises remotely over telehealth to ensure accuracy. The video is then sent to the clinician dashboard for diagnosis and interpretation. In cases of BPPV, clients are then able to use the short arm head mount device and guided video tutorial to ensure correct movements are undertaken during a CRM with assistance from a support person.

\subsection{Challenges}

During the exploration phase, challenges arose around the design and functioning of the mobile application. Using real time video of client's eyes during objective testing in the mobile application was initially suggested. However, this requires high quality internet connection, which is not always available. Therefore, the mobile application was designed to upload the video as soon as the assessment has been completed. Although this may lead to a slightly lengthier consultation session compared to in-person assessment, it aims to provide more accurate diagnostics for telehealth consults whilst removing the time and cost burden of attending a specialist clinic in person. MOVE-IT mobile application uses role models in its demonstration videos and guided video tutorials. As an alternative, animation could have been used, similar to DizzyFix which is a mobile application that guides medical students through an Epley's maneuverer using a guided animation and gyroscope data to ensure correct head position [8]. Animations were not chosen in the development of MOVE-IT as this would have posed additional challenges, increasing costs of development and a lengthier production time.

Several challenges arose during the experimentation phase, when attempting to produce a video clear enough to identify nystagmus and other oculomotor impairments from a smart phone that is moving whilst recording. During testing the head mount device was found to be stable when the head position was upright. However, when the client was required to extend their neck or lie down the head mount device would slip upwards and occasionally off the head completely. To address this issue, an additional strap was added under the chin to prevent significant movement during BPPV testing. Another challenge arose when testing MOVE-IT with CRMs. CRMs require a sequence of various head positions resulting in the arm of the head mount hitting the bed. As visuals of the eyes is not necessarily required during CRMs, this issue was solved by adding an interchangeable short arm to the head mount device to allow the mobile device to be placed closer to the forehead when performing CRMs, to avoid the device getting in the way of the maneuverer. 


\subsection{Strengths and limitations}

A collaborative process was used, involving end-users and stakeholders in a real-life environment, with the aim to reduce challenges and issues in the final product. Scoping previous literature and obtaining clinician perspectives through the focus group and consultation interviews provided insight into prior successes in this area and possible challenges. We developed the mobile application for both Android and IOS, and designed the low-cost head mount to hold most types of phones, improving accessibility.

MOVE-IT is limited in that it relies on a support person with likely little to no experience in vestibular or oculomotor assessment or CRMs. This may result is lower accuracy of assessments compared to an experienced clinician completing the physical assessment in person. Face-to-face assessments allow clinicians to take individuals from an assessment straight into treatment. This reduces the amount of times the client's dizziness is provoked. As use of real-time video was not feasible in this system, individuals are required to complete the assessment, send the recorded video through to the clinician and then complete the appropriate CRM as guided by the clinician, leading to a slightly lengthier process.

\section{Conclusion \& Future Directions}

The living Lab method utilized in this study has been successful in the exploration and experimentation phase of the development of MOVE-IT. MOVE-IT meets all predefined functionality requirements identified in the exploration phase. Once the evaluation phase is complete, we envisage that MOVE-IT will enable remote vestibular assessment and treatment using telehealth in the concussion population. Future studies will start by investigating the validity of MOVE-IT in clinical testing. This will be followed by usability testing with clients and a support person onsite to identify and address potential issues. Following these stages, MOVE-IT will be tested via telehealth with clients offsite in a real-life setting. Following successful testing, MOVE-IT would prove advantageous for vestibular and oculomotor assessments and BPPV treatments for a range of circumstances including individuals in rural and remote areas, individuals unable to tolerate car trips to onsite clinicians, areas limited by COVID-19 quarantining restrictions, assisting less experienced clinicians from offsite locations and sporting teams when interstate or overseas. MOVE-IT may also be extended past the concussion population to other vestibular impairment populations.

\section{References}

1. Thomas E, Fitzgerald M, Cowen G. Post-concussion states: How do we improve our patients' outcomes? An Australian perspective. Journal of Concussion. 2020;4:1-4.

2. Ellis MJ, Leddy J, Cordingley D, Willer B. A physiological approach to assessment and rehabilitation of acute concussion in collegiate and professional athletes. Frontiers in neurology. 2018;9:1115.

3. Wallace B, Lifshitz J. Traumatic brain injury and vestibulo-ocular function: current challenges and future prospects. Eye and brain. 2016;8:153.

4. Gurley JM, Hujsak BD, Kelly JL. Vestibular rehabilitation following mild traumatic brain injury. NeuroRehabilitation. 2013;32(3):519-28.

5. Snoswell CL, Caffery LJ, Haydon HM, Thomas EE, Smith AC. Telehealth uptake in general practice as a result of the coronavirus (COVID-19) pandemic. Australian Health Review. 2020;44(5):737-40. 
6. Schulz T, Long K, Kanhutu K, Bayrak I, Johnson D, Fazio T. Telehealth during the coronavirus disease 2019 pandemic: Rapid expansion of telehealth outpatient use during a pandemic is possible if the programme is previously established. Journal of Telemedicine and Telecare. 2020:1-7.

7. Nussbaum R, Kelly C, Quinby E, Mac A, Parmanto B, Dicianno BE. Systematic review of mobile health applications in rehabilitation. Archives of physical medicine and rehabilitation. 2019;100(1):115-27.

8. Organ B, Liu H, Bromwich M. An iPhone-assisted particle repositioning maneuver for benign paroxysmal positional vertigo (BPPV): a prospective randomized study. The Journal of the American Board of Family Medicine. 2015;28(1):118-20.

9. Meldrum D, Skinner R, Molina B. Vertigenius [Available from: www.vertigenius.com].

10. Shah MU, Lotterman S, Roberts D, Eisen M. Smartphone telemedical emergency department consults for screening of nonacute dizziness. The Laryngoscope. 2019;129(2):466-9.

11. Kıroğlu M, Dağkıran M. The Role of Mobile Phone Camera Recordings in the Diagnosis of Meniere's Disease and Pathophysiological Implications. The Journal of International Advanced Otology. 2020;16(1):18.

12. Kim J, Kim YL, Jang H, Cho M, Lee M, Kim J, et al. Living labs for health: an integrative literature review. European Journal of Public Health. 2020;30(1):55-63.

13. Evans P, Schuurman D, Stahlbrost A, Vervoort K. Living Lab Methodology Handbook 2017.Available from: https://www.ltu.se/cms_fs/1.101555!/file/LivingLabsMethodologyBook_web.pdf

14. Feurstein K, Hesmer A, Hribernik KA, Thoben K, Schumacher J. Living Labs: a new development strategy. European Living Labs-a new approach for human centric regional innovation. 2008:1-14.

15. Martinez RN, Hogan TP, Lones K, Balbale S, Scholten J, Bidelspach D, et al. Evaluation and treatment of mild traumatic brain injury through the implementation of clinical video telehealth: Provider perspectives from the Veterans Health Administration. PM\&R. 2017;9(3):231-40.

16. Caze II T, Knell GP, Abt J, Burkhart SO. Management and Treatment of Concussions via TeleConcussion in a Pediatric Setting: Methodological Approach and Descriptive Analysis. JMIR Pediatrics and Parenting. 2020;3(2):e19924 